\title{
ASPECTOS ULTRA-SONOGRÁFICOS DA NEOPLASIA EPITELIAL PAPILÍFERA SÓLIDO-CÍSTICA DO PÂNCREAS*
}

\author{
Márcio Martins Machado ${ }^{1}$, Ana Cláudia Ferreira Rosa ${ }^{2}$, Nestor de Barros ${ }^{3}$, Marcel C.C. \\ Machado $^{4}$, Letícia Martins Azeredo ${ }^{5}$, Luciana Mendes de Oliveira Cerri ${ }^{6}$, Orlando Milhomem \\ da Mota ${ }^{7}$, Jales Benevides Santana Filho ${ }^{8}$, Paulo Moacir de Oliveira Campoli ${ }^{8}$, Daniela \\ Medeiros Milhomem Cardoso ${ }^{9}$, Giovanni Guido Cerri ${ }^{10}$
}

Resumo Os autores relatam os aspectos clínicos, macroscópicos e ultra-sonográficos em 11 pacientes com neoplasia epitelial papilífera sólido-cística (NEPSC) de pâncreas avaliados prospectivamente. Dez pacientes eram do sexo feminino e um era do sexo masculino, com idades entre 15 e 75 anos (média de 27 anos). Todos os pacientes tiveram diagnóstico de NEPSC de pâncreas comprovado por exame histopatológico e foram submetidos a exames de ultra-sonografia no pré-operatório. Foram avaliadas as dimensões, a localização e os aspectos ultra-sonográficos dos tumores, realizando-se correlação com suas características macroscópicas. $O$ diâmetro transverso médio das lesões foi de 8,7 cm (variação: 4,2 a 16,0 cm). Três localizavam-se na cabeça, três no corpo e cinco na cauda do pâncreas. Todas as lesões tinham algum grau de hemorragia intratumoral e eram bem delimitadas e encapsuladas. Em todos os casos houve correlação completa entre o aspecto macroscópico dos tumores com os achados da ultra-sonografia. Os tumores eram predominantemente sólidos em três casos $(27,3 \%)$, císticos em um $(9,1 \%)$ e com aspecto sólido-cístico em sete $(63,6 \%)$. Foram identificadas calcificações, predominantemente periféricas, em quatro $(36,4 \%)$ dos 11 pacientes. Todos os tumores estabeleciam contato com algum segmento do eixo venoso espleno-mesentérico-portal. Em apenas um caso foi confirmada invasão vascular tumoral. Não foram identificadas metástases ganglionares ou a distância em nenhum paciente. Os autores concluem que as características ultra-sonográficas se correlacionaram com os aspectos macroscópicos das lesões em todos os casos, e que, embora não-específicas, elas são bastante sugestivas do diagnóstico de NEPSC se analisadas no contexto clínico apropriado. Unitermos: Neoplasia de pâncreas; Neoplasia epitelial papilífera sólido-cística de pâncreas; Ultra-sonografia.

Abstract Ultrasonography findings of solid-cystic papillary epithelial neoplasm of the pancreas.

The authors report the clinical, gross pathology and ultrasonography findings of 11 prospectively studied patients with solid and papillary epithelial neoplasm of the pancreas (SPEN). The patients were one male and ten females aged 15 to 75 years (mean age at diagnosis: 27 years). All patients had pathology proven SPEN and were submitted to preoperative ultrasonography. The size, location and ultrasonographic features of the tumors were evaluated and correlated with the gross pathology findings. The mean transverse diameter of these tumors was $8.7 \mathrm{~cm}$ (range: $4.2 \mathrm{~cm}$ to $16.0 \mathrm{~cm}$ ). Tumors were located in the head (three cases), body (three cases) and tail (five cases) of the pancreas. All lesions were well encapsulated and presented some degree of internal hemorrhage. There was full correlation between ultrasonography and gross pathology findings. In three cases $(27.3 \%)$ the tumors were predominantly solid, cystic in one case $(9.1 \%)$, and had equal degrees of solid and cystic components in seven cases (63.6\%). Calcifications, predominantly peripheral, were seen in four $(36.4 \%)$ of the 11 patients. All tumors were in contact with the portal, mesenteric and splenic venous axis. In all cases except one there was no venous invasion. Lymph nodes or distant metastases were not observed in any of the patients. The authors concluded that in all cases there was a correlation between the ultrasonography and gross pathology findings, and although ultrasonography findings are not specific they may be highly suggestive of SPEN in the appropriate clinical context.

Key words: Neoplasm of the pancreas; Solid and papillary epithelial neoplasm of the pancreas; Ultrasonography.

\footnotetext{
* Trabalho realizado nos Departamentos de Radiologia do Hospital das Clínicas da Faculdade de Medicina da Universidade de São Paulo (HC-FMUSP) e do Hospital Sírio Libanês, São Paulo, SP, e no Departamento de Doenças do Aparelho Digestivo do Hospital Araújo Jorge (Hospital do Câncer) da Associação de Combate ao Câncer em Goiás (ACCG), Goiânia, GO.

1. Médico Radiologista do Hospital das Clínicas da Faculdade de Medicina da Universidade Federal de Goiás (HC-FMUFG) Médico Radiologista Consultor do Departamento de Doenças do Aparelho Digestivo do Hospital Araújo Jorge (Hospital do Câncer) da ACCG, Chefe da Divisão de Radiologia Músculo-Esquelética e de Emergência do Centro de Diagnóstico do Hospita de Acidentados de Goiânia, Doutor em Radiologia pela FMUSP.

2. Médica Radiologista do HC-FMUFG e da Divisão de Radio-
}

logia Músculo-Esquelética e de Emergência do Centro de Diagnóstico do Hospital de Acidentados de Goiânia, Doutora em Radiologia pela FMUSP.

3. Professor Doutor do Departamento de Radiologia da FMUSP. 4. Professor Titular da Disciplina de Transplante de Fígado e Cirurgia Experimental, Chefe do Senviço de Pâncreas e Vias Biliares da Disciplina de Cirurgia do Aparelho Digestivo da FMUSP. 5. Médica Ultra-sonografista do Serviço de Ultra-Sonografia do Hospital Mater Dei e do Hospital São Francisco, Belo Horizonte, MG.

6. Médica Radiologista Assistente Doutora do Instituto de Radiologia (InRad) do HC-FMUSP, Chefe do Setor de Ultra-Sonografia da Divisão de Clínica Urológica do HC-FMUSP.

7. Chefe e Titular do Departamento de Doenças do Aparelho
Digestivo do Hospital Araújo Jorge (Hospital do Câncer) da ACCG. 8. Médicos Titulares do Departamento de Doenças do Aparelho Digestivo do Hospital Araújo Jorge (Hospital do Câncer) da ACCG.

9. Médica Residente do Departamento de Cirurgia do HCFMUFG.

10. Professor Titular do Departamento de Radiologia da FMUSP, Chefe do InRad/HC-FMUSP, Diretor do Centro de Diagnóstico do Hospital Sírio Libanês.

Endereço para correspondência: Dr. Márcio Martins Machado. Rua 1027 ํo 230, Ed. Fabiana, apto. 304. Goiânia, GO, 74823-120. E-mail: marciommachado@ibest.com.br

Recebido para publicação em 31/10/2002. Aceito, após revisão, em 22/1/2003. 


\section{INTRODUÇÃO}

Os adenocarcinomas pancreáticos correspondem a tumores agressivos, com grande potencial de invasão local e vascular, sendo as metástases a distância achado comum em suas evoluções ${ }^{(\mathbf{1})}$. Outros tumores pancreáticos apresentam comportamento menos agressivo, dentre estes a neoplasia epitelial papilífera sólido-cística do pâncreas (NEPSC). Estes são tumores raros e de baixa agressividade, encontrados usualmente em mulheres jovens ${ }^{(2-12)}$. A NEPSC, ou tumor de Frantz, foi descrita pela primeira vez por Frantz em $1959^{(13)}$, e ocorreria predominantemente na segunda e terceira décadas de vida.

No presente estudo os autores apresentam as características encontradas em 11 casos de NEPSC, assim como seus aspectos de apresentação ultra-sonográfica.

\section{MATERIAL E MÉTODOS}

Foram avaliados, prospectivamente, 11 pacientes portadores de NEPSC, no período de 1994 a 2002, advindos dos Departamentos de Radiologia do Hospital das Clínicas da Faculdade de Medicina da Universidade de São Paulo e do Hospital Sírio Libanês, São Paulo, SP, e do Departamento de Doenças do Aparelho Digestivo do Hospital Araújo Jorge (Hospital do Câncer) da Associação de Combate ao Câncer em Goiás, Goiânia, GO. Os achados de ultra-sonografia (US) foram comparados com os obtidos durante as cirurgias e pelo exame anatomopatológico, conforme padronização estabelecida no início do período do estudo e descrita a seguir.

A idade dos pacientes variou de 15 a 65 anos (média de 27 anos). Dez pacientes eram do sexo feminino e um era do sexo masculino. Três eram brancos $(27,3 \%)$, três eram amarelos $(27,3 \%)$, três eram negros $(27,3 \%)$ e dois eram pardos $(18,1 \%)$. Com relação aos sintomas clínicos, oito $(72,7 \%)$ pacientes apresentaram dor abdominal difusa, cinco $(45,4 \%)$ tinham massa palpável e três $(27,3 \%)$ pacientes tiveram o tumor identificado durante exame de US abdominal por outra causa.

Os exames de US abdominal foram realizados com aparelho Logic 500 ou Logic 700 (GE, Milwaukee, Wisconsin, USA),
Toshiba SSH-140 e SSD-500 (Tóquio, Japão) e Sonoline-Elegra-Siemens Medical Systems (Issaquah, Washington, USA).

A glândula pancreática foi examinada sempre nos planos transversal, longitudinal e oblíquos, de maneira dinâmica, compreendendo estudo de todos os seus eixos de coordenadas, possibilitando um estudo de todas as relações das lesões com as estruturas adjacentes, permitindo uma percepção volumétrica dessas lesões. Durante o exame procurou-se avaliar criteriosamente a ecotextura das lesões e suas dimensões e relações com o eixo venoso espleno-mesentérico-portal. Com relação a este último aspecto, avaliou-se apenas se a lesão estabelecia inequívoco contato com o vaso venoso adjacente ou não.

A ecotextura foi comparada com aquela do pâncreas normal. Do ponto de vista ultra-sonográfico, as áreas sólidas foram consideradas como sendo as que apresentavam ecotextura hipo ou isoecogênica. No caso de áreas sólidas hipoecogênicas, não se observaram ecos em suspensão em maior ou menor grau. As áreas heterogêneas hipoecogênicas, isoecogênicas e hiperecogênicas (sem sombra acústica posterior) também foram consideradas como sendo componentes sólidos. As áreas císticas corresponderiam àquelas apresentando ecotextura anecóica ou hipoecogênica, com "ecos em suspensão", em maior ou menor grau. As áreas hiperecogênicas com sombra acústica posterior foram consideradas como sendo decorrentes de calcificações.

Não foi estabelecido critério específico para a determinação do componente hemorrágico-necrótico. Na opinião dos autores do presente trabalho e de outros autores, na literatura ${ }^{(\mathbf{1 0 )}}$, o componente cístico da NEPSC é decorrente, sobretudo, dos processos de hemorragia e necrose, sendo então avaliados pela identificação do próprio componente cístico. A presença de ecos em suspensão indica líquido espesso no interior dos cistos, que nestes tumores representam as próprias alterações involutivas necrótico-hemorrágicas.

Embora de modo arbitrário, consideraram-se as lesões como sólidas, sólido-císticas e císticas, segundo os critérios ultrasonográficos descritos acima. Quando eram amplamente sólidas, foram nomeadas de sólidas, embora em algumas situações o exame macroscópico tenha evidenciado diminutas áreas focais de hemorragia intratumoral, sem contudo constituírem áreas de aspecto cístico. No caso de apresentarem componentes císticos, associados a áreas sólidas, foram denominadas de sólido-císticas. E aquelas que se mostravam amplamente císticas foram denominadas de císticas. Todas as lesões foram cuidadosamente submetidas a finos cortes macroscópicos (4 mm de espessura), para confirmação ou não da correlação entre a macroscopia e os achados ultra-sonográficos.

Em todos os casos foi feita a comparação entre os achados ultra-sonográficos, os da macroscopia cirúrgica e os da macroscopia ex-vivo, sempre com a presença de um dos cirurgiões envolvidos diretamente na cirurgia, do patologista e de um membro da equipe de diagnóstico por imagem.

Foi também determinado o diâmetro transverso máximo dos tumores, tendo como medida definitiva o resultado desta mensuração ex-vivo, após a retirada da peça cirúrgica.

Em cinco casos realizou-se também ultra-sonografia intra-operatória (USIO) das lesões e do fígado (neste caso, para investigação de possíveis metástases). Dois dos presentes casos foram motivo de publicações anteriores $^{(4,8)}$. A metodologia utilizada pelos autores para a realização da USIO já foi previamente publicada ${ }^{(\mathbf{1 4}, \mathbf{1 5})}$.

\section{RESULTADOS}

Três lesões $(27,3 \%)$ localizavam-se na cabeça do pâncreas, três $(27,3 \%)$ no corpo e cinco $(45,4 \%)$ na cauda. Com relação às dimensões das lesões, o diâmetro máximo variou de 4,2 a $16 \mathrm{~cm}$ (média de $8,7 \mathrm{~cm}$ ).

Houve correlação concordante em todos os casos entre os critérios ultra-sonográficos para definição dos componentes do tumor (componente sólido, cístico e presença de calcificações) e os aspectos macroscópicos da lesão. Dessa maneira, dos 11 pacientes, dois $(18,2 \%)$ apresentaram tumores predominantemente sólidos, sete $(63,6 \%)$ tinham tumores sólido-císticos e um $(9,1 \%)$ era predominantemente cístico, sem nenhum componente sólido periférico identificado à macroscopia (embora houvesse células neoplásicas na parede do cisto). Esta lesão predominante- 
mente cística apresentava um septo espesso representando predominantemente tecido necrótico-hemorrágico, embora houvesse, de permeio, algumas células neoplásicas (Figura 1). As calcificações estiveram presentes em quatro pacientes $(36,4 \%)$, com distribuição predominantemente periférica em todos, tendo sido identificadas pela US na totalidade dos casos. Foram identificadas áreas hemorrágicas intratumorais em todos os casos pelo exame anatomopatológico.

Em todas as lesões, provavelmente devido às suas grandes dimensões, a US identificou contato da lesão com pelo menos um dos vasos venosos do eixo venoso espleno-mesentérico-portal. Não foi observada trombose no referido eixo venoso em nenhuma eventualidade, também não foi observada invasão tumoral interrompendo a estrutura da parede venosa ao ultra-som.

Em um paciente, durante a cirurgia, foi observada aderência firme entre o tumor e a junção venosa mesentérico-portal. $\mathrm{O}$ tumor foi ressecado com um pequeno segmento venoso neste ponto. Foi confirmada invasão focal microscópica neste ponto pelo exame histopatológico da peça cirúrgica, por uma extensão de $0,4 \mathrm{~cm}$ na junção mesentérico-portal.

Não houve casos de metástases linfonodais, implantes peritoneais ou de metástases a distância (hepáticas ou extra-hepáticas), identificadas no momento da cirurgia. Embora todos os casos ainda estejam em acompanhamento, os achados deste acom- panhamento serão motivo de futura publicação, não sendo abordados neste estudo.

Todos os pacientes foram submetidos a intervenção cirúrgica, com remoção completa da lesão, e os pacientes tiveram alta hospitalar em ótimas condições clínicas. $\mathrm{O}$ diagnóstico histopatológico de todos os casos foi de NEPSC (tumor de Frantz).

\section{DISCUSSÃO}

A NEPSC é uma neoplasia pancreática incomum. Entretanto, merece atenção especial, pois tem características clínicas, morfológicas e de imagem que auxiliam no seu diagnóstico. Embora esses achados não sejam específicos dessa neoplasia, no contexto clínico específico, considerando os

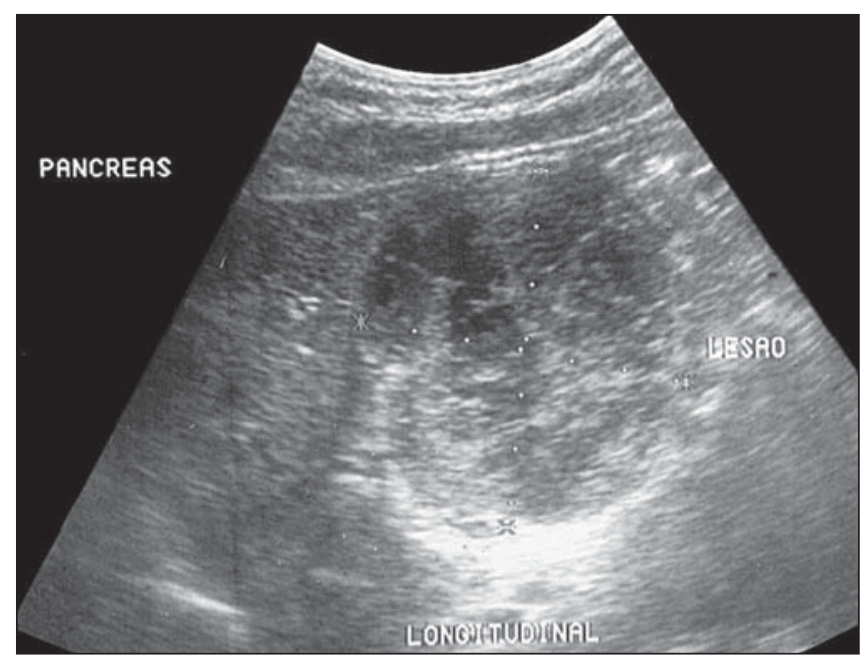

A

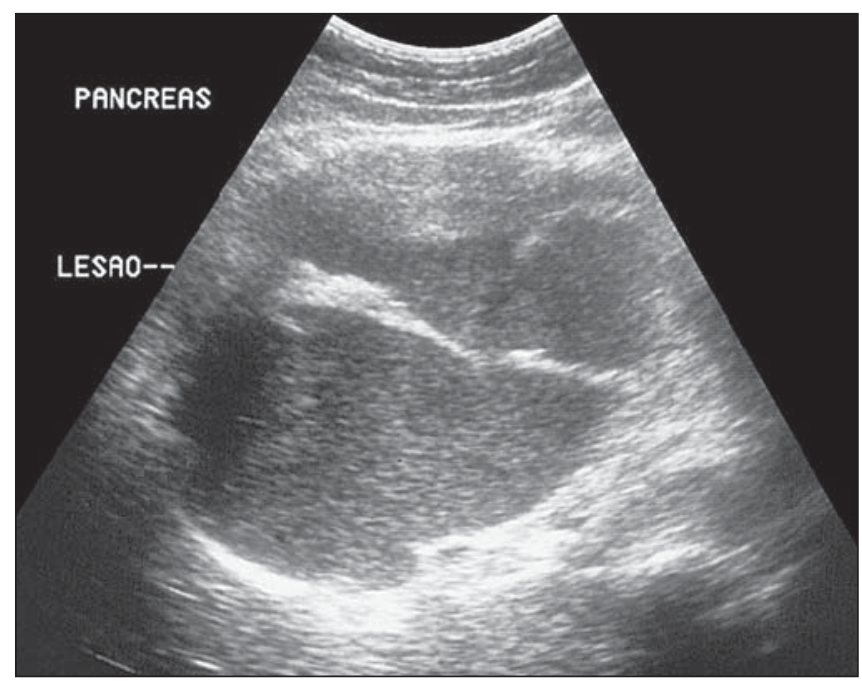

C

Figura 1. Neoplasia epitelial papilífera sólido-cística de pâncreas. A,B: Lesão sólido-cística. C: Lesão predominantemente cística, apresentando septo espesso. D: Lesão predominantemente sólida.

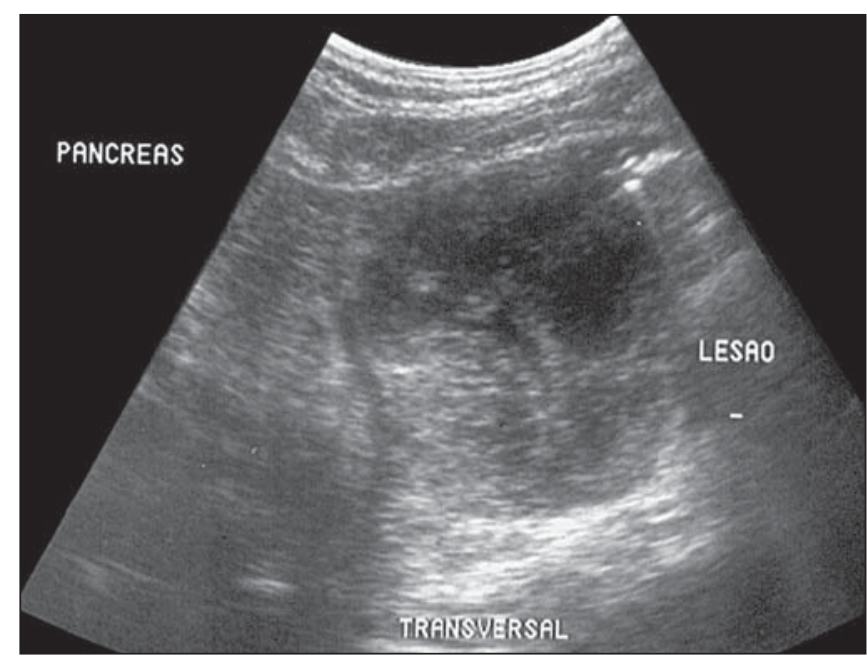

B

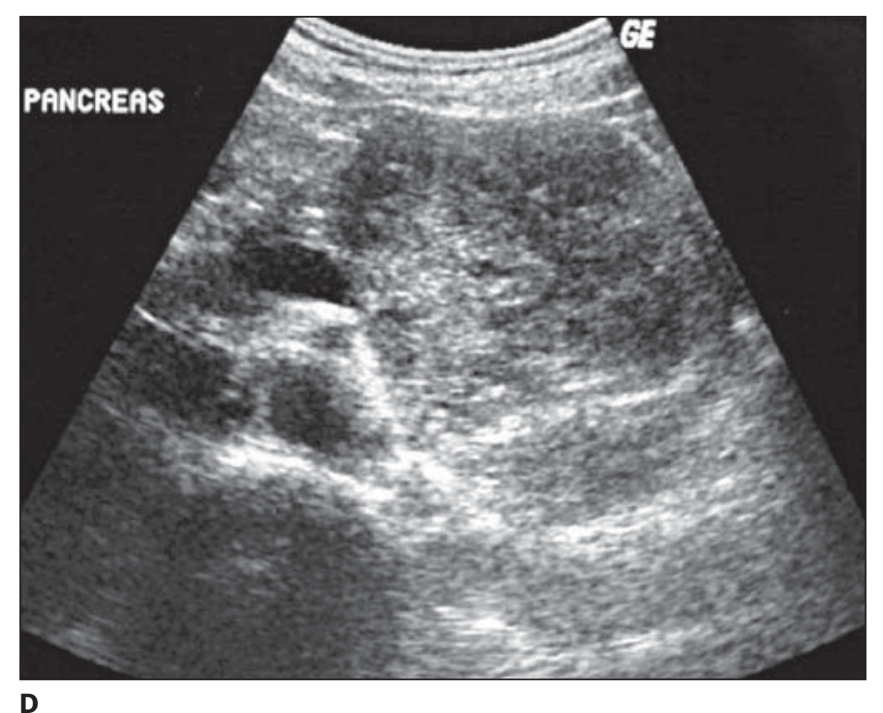


aspectos epidemiológicos e morfológicos desses tumores pode-se chegar a um diagnóstico presuntivo em muitos $\operatorname{casos}^{(1-18)}$.

A NEPSC representa uma variante de tumor com baixo potencial de agressividade (malignidade). Não obstante, deve ser removida cirurgicamente, para que se possa obter a cura do paciente ${ }^{(\mathbf{1 9 , 2 0})}$. A maioria dos pacientes operados não apresenta recidiva da lesão, quer regionalmente ou a distância ${ }^{(20)}$. Contudo, casos de metástases regionais e a distância têm sido descritos na literatura ${ }^{(\mathbf{1 0 , 2 1})}$.

A cirurgia, embora curativa, apresenta graus variados de dificuldade técnica em sua execução, diante das dimensões desses tumores, que comumente estão adjacentes a segmentos vasculares importantes, particularmente os venosos ${ }^{(\mathbf{2 , 4 , 8 )}}$. Embora não haja invasão vascular na maioria dos casos, do ponto de vista tático-cirúrgico, a liberação do tumor dos vasos adjacentes demanda muita habilidade e familiaridade com a cirurgia pancreática ${ }^{(2)}$. Porém, não podem ser esquecidos os casos incomuns de presença de invasões vasculares ${ }^{(\mathbf{1 0 , 2 1})}$.

Do exposto, fica evidente o papel fundamental do médico radiologista ou ultrasonografista, que realizando um exame meticuloso poderá já fornecer indícios dos locais na anatomia regional onde as compressões vasculares seriam mais evidentes, antecipando possíveis dificuldades táticas na cirurgia. Utilizando essas informações, os cirurgiões poderiam se orientar melhor durante a ressecção dessas lesões.

Nos nossos casos todas as lesões apresentavam contato com pelo menos um segmento do eixo venoso espleno-mesentérico-portal. Consideramos este fato decorrente das grandes dimensões dessas lesões (média de $8,7 \mathrm{~cm}$, variando de $4,2 \mathrm{~cm}$ a $16,0 \mathrm{~cm}$ ) na ocasião do diagnóstico. Essas dimensões, no presente estudo, correspondem àquelas referidas na literatura ${ }^{(22)}$. Em todos os nossos casos as relações vasculares dos tumores com os vasos adjacentes, demonstradas pela US, foram correspondentes aos achados cirúrgicos. Em apenas um caso havia invasão focal $(0,4 \mathrm{~cm})$ na junção venosa mesentérico-portal (que não foi identificada pela US), suspeitada durante a cirurgia e confirmada pelo exame histopatológico do pequeno segmento venoso ressecado juntamente com o tumor, na tentativa de se obter a radicalidade oncológica. Este fato, isoladamente, não compromete as contribuições do estadiamento ultra-sonográfico, na medida em que o exame de US demonstrou topograficamente os pontos de contato do tumor com os vasos adjacentes, em todos os casos e inclusive neste de invasão. Os cirurgiões sabem que, na NEPSC, o contato com os vasos não significa invasão vascular na grande maioria dos casos. Ademais, há muito é reconhecida a limitação dos métodos de imagem (US, tomografia computadorizada (TC) convencional ou helicoidal e ressonância magnética) na identificação de pequenas áreas focais de invasão vascular, em especial em neoplasias que apenas raramente

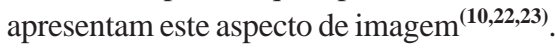

Com relação às características patológicas, essas lesões se caracterizam por serem bem delimitadas e encapsuladas. Possuem áreas sólidas contendo delicada e numerosa rede de vasos sanguíneos, identificando-se, também, áreas císticas e papilares. A predominância destes componentes depende da intensidade dos fenômenos de hemorragia e degeneração (necrose) que ocorrem nesses tumores. As alterações hemorrágicas no interior das lesões são muito comuns ${ }^{(\mathbf{1 0 , 1 9 )}}$, tendo sido identificadas em 55 dos 56 casos avaliados por Buetow et al. ${ }^{(\mathbf{1 0})}$. As calcificações podem ocorrer, apresentando padrão periférico, sendo identificadas em cerca de $30 \%$ dos casos, segundo alguns autores ${ }^{(\mathbf{1 0})}$. As metástases linfonodais, hepáticas e peritoneais, e a invasão vascular e de órgãos adjacentes, embora pouco comuns, têm sido referidas na literatura ${ }^{(\mathbf{1 0 , 2 1 )}}$.

Nos nossos casos, as áreas de hemorragia no interior do tumor foram identificadas em todos os casos, enquanto as calcificações foram observadas em $36,4 \%$ dos pacientes. Chama-nos a atenção o fato de alguns autores ${ }^{(\mathbf{1 0})}$ não terem sido capazes de identificar calcificações pela US em nenhum caso, tendo sido todas as calcificações identificadas apenas pela TC. Na nossa casuística, identificamos com facilidade todos os casos com calcificação por meio da US, havendo concordância completa com o resultado do exame anatomopatológico. De conformidade com outros autores $^{(\mathbf{1 0})}$, todas as calcificações tinham distribuição predominantemente periférica.
No estudo de Buetow et al. ${ }^{(\mathbf{1 0})}$, em 20 casos $(35,7 \%)$ as lesões eram predominantemente sólidas, em 14 (25\%) eram predominantemente císticas e em $22(39,3 \%)$ eram sólido-císticas. Nos nossos casos, somente três $(27,3 \%)$ lesões eram predominantemente sólidas, uma $(9,1 \%)$ era cística e sete $(63,6 \%)$ eram sólido-císticas (Figura 1). Essas diferenças poderiam ser decorrentes da diferença no número de casos analisados nos dois estudos, e também devidas às diferenças metodológicas adotadas para nomear as lesões como sólidas, císticas e sólido-císticas.

A NEPSC acomete preferencialmente o sexo feminino em idade jovem, predominando nos negros e amarelos ${ }^{(\mathbf{1 0 , 1 2})}$. No presente estudo também tivemos amplo predomínio de acometimento do sexo feminino (só um caso era do sexo masculino). Com relação ao grupo étnico, nosso país é bastante miscigenado com a raça negra, e juntamente com o pequeno número de casos de nosso estudo não permite amplas correlações neste sentido. $\mathrm{O}$ fato de termos três orientais entre os nossos casos pode até representar tendência maior do acometimento deste grupo étnico, mas esta informação deve ser analisada com cautela, tendo em vista as limitações referidas anteriormente em relação ao nosso estudo.

A idade média dos nossos pacientes, na ocasião do diagnóstico, foi de 27 anos, também estando de conformidade com os dados da literatura ${ }^{(\mathbf{1 0 , 1 3})}$, que referem acometimento preferencial na segunda e terceira décadas de vida. De qualquer forma, conforme referido por outros estudos ${ }^{(\mathbf{1 0 - 1 2})}$ e também visto no nosso estudo, a NEPSC pode acometer qualquer raça e idades variadas, desde pacientes tão jovens quanto dez anos de idade até idades mais avançadas, como 75 anos.

Na presente casuística três lesões localizavam-se na cabeça $(27,3 \%)$, três situavam-se no corpo $(27,3 \%)$ e cinco localizavam-se na cauda $(45,4 \%)$ do pâncreas. Dessa forma, $72,7 \%$ dos tumores encontravam-se no corpo e na cauda do pâncreas. Outros autores também encontraram esta predominância $^{(\mathbf{1 0 - 1 2})}$.

As características clínicas dos pacientes deste estudo, como o número significativo de pacientes com dores abdominais vagas e difusas, massa palpável e mesmo 
pacientes sem qualquer sintoma clínico ou sinal físico, correspondem àquelas de outros estudos ${ }^{(\mathbf{1 1}, \mathbf{1 2})}$.

No conhecimento dos autores, no Brasil, os primeiros casos de NEPSC estudados do ponto de vista clínico, cirúrgico e com aspectos de imagem foram descritos por Machado et al. ${ }^{\text {(2) }}$, em 1993. Ainda com relação aos métodos de imagem, foram Machado et al. ${ }^{(\mathbf{8})}$, em 1999, quem primeiro publicaram suas características à USIO.

Os autores ponderam que avaliações que procuram correlacionar aspectos macroscópicos com achados de imagem (no caso, a US), como aquela utilizada no presente estudo, estão sujeitas a interpretações algo subjetivas, pois comparam imagens obtidas in vivo, com dados macroscópicos obtidos ex-vivo. Portanto, mesmo que se tenha adotado, neste estudo, uma forma de avaliação "lado-a-lado" entre cirurgiões, patologistas e membros da equipe de diagnóstico por imagem, podem existir variações na interpretação dessas correlações.

\section{CONCLUSÃO}

A NEPSC de pâncreas apresenta aspectos clínicos, morfológicos e ultra-sonográficos que, embora não-específicos, podem levar a um diagnóstico presuntivo desses tumores, quando avaliados no contexto clínico apropriado.

\section{REFERÊNCIAS}

1. Murr MM, Sarr MG, Oishi AJ, van Heerden JA. Pancreatic cancer. CA Cancer J Clin 1994; 44:304 18.
2. Machado MCC, Cunha JEM, Bacchella T, et al. Tumor de Frantz (neoplasia epitelial papilar e cística de pâncreas). Estudo de três casos. Rev Hosp Clin Fac Med São Paulo 1993;48:29-34.

3. Machado MM, Rosa ACF, Barros N, et al. Análise evolutiva e perspectiva histórica da ultra-sonografia intra-operatória (USIO) nas afecções pancreáticas. Radiol Bras 2003;36:41-5.

4. Machado MM, Rosa ACF, Barros N, Machado MCC, Cerri GG. Qual o seu diagnóstico? (Neoplasia epitelial papilífera sólido-cística de pâncreas). Radiol Bras 2002;35(2):V-VII.

5. Machado MM, Rosa ACF, Cerri GG. Ultra-sonografia intra-operatória. In: Cerri GG, Oliveira IRS, eds. Ultra-sonografia abdominal. Rio de Janeiro, RJ: Revinter, 2002:573-84.

6. Machado MM, Rosa ACF, Barros N, Machado MCC, Cerri GG. Ultra-sonografia intra-operatória (USIO) do pâncreas e das vias biliares. Radiol Bras 2002;35:105-8.

7. Machado MM, Rosa ACF, Cerri GG. Métodos diagnósticos por imagem. In: Matos AA, Dantas W, eds. Compêndio de hepatologia. $2^{\underline{a}}$ ed. São Paulo, SP. Fundo Editorial BYK, 2001:87-113.

8. Machado MM, Saito OC, Oliveira IRS, Penteado S, Machado MCC, Cerri GG. Tumor de Frantz: características anátomo-sonográficas à ultra-sonografia intra-operatória (USIO) e implicações no manuseio cirúrgico. Nota prévia. Radiol Bras 1999;32:89_ 92.

9. Neto MJF, Machado MM, Oliveira IRS, Cerri GG. Pâncreas. In: Cerri GG, Oliveira IRS, eds. Ultrasonografia abdominal. Rio de Janeiro, RJ: Revinter, 2002:261-94.

10. Buetow PC, Buck JL, Pantongrag-Brown L, Beck KG, Ros PR, Adair CF. Solid and papillary epithelial neoplasm of the pancreas: imaging-pathologic correlation in 56 cases. Radiology 1996;199:70711.

11. Friedman AC, Lichtenstein JE, Fishman EK, Oertel JE, Dachman AH, Siegelman SS. Solid and papillary epithelial neoplasm of the pancreas. Radiology 1985;154:333-7.

12. Ebisui S, Kawamoto K, Mitusfuji S. A case report of solid and cystic tumor (SCT) of the pancreas and literature review of 126 cases of Japan. Gastroenterol Endosc 1990;32:1692-701.

13. Frantz UK. Tumors of the pancreas. Atlas of tumor pathology. Section 7, Fascicles 27 and 28. Washington, DC: Armed Forces Institute of Pathology, 1959:32-3.

14. Cerri LMO. Contribuição da ultra-sonografia intraoperatória em fígado, vias biliares e pâncreas. (Tese de Doutorado). São Paulo, SP: Universidade de São Paulo, 1995.

15. Cerri LMO, Cerri GG. Intraoperative ultrasonography of liver, bile ducts and pancreas. Rev Paul Med 1996;114:1196-207.

16. Lewandrowski KB, Southern JF, Pins MR, Compton CC, Warshaw AL. Cystic fluid analysis in the differential diagnosis of pancreatic cysts. A comparison of pseudocysts, serous cystadenomas, mucinous cystic neoplasms, and mucinous cystadenocarcinoma. Ann Surg 1993;217:41-7.

17. Rubin D, Warshaw AL, Southern JF, Pins M, Compton CC, Lewandrowski KB. Expression of CA 15.3 protein in the cyst contents distinguishes benign from malignant pancreatic mucinous cystic neoplasms. Surgery 1994;115:52-5.

18. Alles AJ, Warshaw AL, Southern JF, Compton CC Lewandrowski KB. Expression of CA 72-4 (TAG72 ) in the fluid contents of pancreatic cysts. A new marker to distinguish malignant pancreatic cystic tumors from benign neoplasms and pseudocysts. Ann Surg 1994;219:131-4.

19. Pettinato G, Manivel JC, Ravetto C, et al. Papillary cystic tumor of the pancreas. A clinicopathologic study of 20 cases with cytologic, immunohistochemical, ultrastructural, and flow cytometric observations, and a review of the literature. Am J Clin Pathol 1992;98:478-88.

20. Zinner MJ, Shurbaji MS, Cameron JL. Solid and papillary epithelial neoplasms of the pancreas. Surgery 1990;108:475-80.

21. Nishihara K, Nagoshi M, Tsuneyoshi M, Yamaguchi K, Hayashi I. Papillary cystic tumors of the pancreas. Assessment of their malignant potential. Cancer 1993;71:82-92.

22. Mathieu D, Guigui B, Valette PJ, et al. Pancreatic cystic neoplasms. Radiol Clin North Am 1989; 27:163-76.

23. Ohtomo K, Furui S, Onoue M, et al. Solid and papillary epithelial neoplasms of the pancreas: MR imaging and pathologic correlation. Radiology 1992;184:567-70. 